ceux!). Deux auteurs proposent trois grands groupes de localisation et de besoins de proximité qui permettent de fonder une définition de trois catégories d'espace : les villes, les espaces ruraux périurbains et les espaces éloignés bien verdoyants. Ne reste plus qu'à voir comment ces derniers peuvent se sentir moins éloignés grâce aux technologies de l'information et de communication. C'est l'objet de l'ouvrage suivant.

\section{Guide TIC des petites et moyennes collectivités : à l'usage des élus, cadres et fonctionnaires territoriaux Paris, FICOME, 2004, 124 p.}

La Fédération interprofessionnelle de la communication d'entreprise (FICOME) souhaite, par ce petit ouvrage, accroître l'efficacité des administrations tout en assurant un accès permanent aux services publics pour les citoyens et les entreprises bien conscients de leurs responsabilités dans le développement économique. Fibre optique ? ASDL ? WiFi ? Courants en ligne ? Voilà autant de questions dont un début de réponse se trouve dans un premier chapitre portant sur l'accès au haut débit. Pour que le lecteur québécois s'y retrouve avec tous ces sigles et expressions d'oùtre-Atlantique, voire d'outre-Manche, en annexe un lexique "numérique » permet d'en découdre avec les ASP, ASDL, BLR, CPL, HFC, IP-PBX, LAN, MAN, PABX et autres UMTS, ou encore WAN, WAP. Quant à WiFi, nous savions tous que ça réfère à Wireless Fidelity que les Montréalais connaissent grâce à leurs cafés Secund Cup.

Pour les rédacteurs de ce guide, le Top 4 des usages pour une petite collectivité se présente ainsi :

a) E-administration : il s'agit de généraliser l'usage du courriel (e-mail dans le texte).

b) Augmenter la qualité de la vie par les points d'accès à l'Internet. Ici, notre beau et grand pays n'a pas de leçons à recevoir.

c) Rendre le territoire plus compétitif par l'accès au haut débit pour les entreprises. Ici, le Canada n'est malheureusement pas le "plus meilleur » pays du monde...

d) Renforcer la sécurité des biens et des personnes en situation de crise. On évoque les inondations, les tremblements de terre, les incendies de forêt... Il est vrai que, si les infortunés de Nouvelle-Orléans avaient été mieux branchés, on aurait pu plus facilement prendre connaissance de leurs malheurs... Mais il ne s'agit pas d'une petite ni même moyenne collectivité.

On revient à nos moutons en considérant la section la plus intéressante pour nos élus et ceux qui gravitent tout autour : le développement économique et l'emploi. On évoque surtout ici les conditions d'accueil de nouvelles entreprises en se rapportant à la technologie qui permet le partage d'équipements télécoms et le partage sécurisé de ressources personnalisées entre différents groupes d'abonnés ou d'entreprises. Ici, on parle d'équipement $\mathrm{PBX}$ (si nécessaire; prière de consulter le lexique en annexe). En fait, comme on le pense bien, tout ce beau monde doit travailler la main dans la main. C'est que démontre la prochaine recension.

\section{"L’inter-communalité » Revue Regards sur l'actualité Paris, La Documentation Française, $n^{\circ} 314$, octobre 2005, 91 p.}

Ce numéro de la revue Regards sur l'actualité porte essentiellement sur un sujet qui fait couler passablement d'encre en France depuis des années. Quand on sait qu'il existe en Hexagone pas moins de 36565 municipalités, dites communes, soit $40 \%$ de ce que compte l'Union européenne à vingt-cinq, ça en fait des maires et des conseillers municipaux, comme dirait le père Ovide. De là l'importance de faire en sorte que tout ce beau monde puisse se parler. Pour ce faire, on a créé exprès un nouveau sigle : EPCI (Établissement public de coopération intercommunale). Quand on connaît l'importance de l'esprit de clocher qui a toujours caractérisé tous les Clochemerles des descendants de nos ancêtres les Gaulois, l’expression « révolution intercommunale » n'est pas trop forte pour souligner le changement apparu dans les années 1990. En effet, à travers les CIDA (oui, vous avez bien lu : Chartes intercommunales de développement et d'aménagement), on apprenait à travailler ensemble plutôt que de toujours se prendre au chignon. Ainsi, petit train va loin, au $1^{\mathrm{er}}$ janvier la fille aînée de l’Église comptait pas moins de 2525 EPCI ressemblant $88 \%$ des communes et $82 \%$ de la population. Félicitations 